九州大学学術情報リポジトリ

Kyushu University Institutional Repository

Reduced high and low frequency gamma synchronization in patients with chronic schizophrenia

\title{
土本，利架子
}

九州大学大学院医学系学府

https://doi.org/10.15017/21718

出版情報：九州大学，2011，博士（医学），課程博士 バージョン：

権利関係: (C) 2011 Elsevier B.V. 


\section{Reduced high and low frequency gamma synchronization in patients with chronic schizophrenia}

\section{(Revision for Schizophrenia Research as a full-length paper)}

Rikako Tsuchimito, M.D. ${ }^{1}$, Shigenobu Kanba, M.D., Ph.D. ${ }^{1}$, Shogo Hirano, M.D., Ph.D. ${ }^{1}$, Naoya Oribe, M.D., Ph.D. ${ }^{1}$, Takefumi Ueno, M.D., Ph.D. ${ }^{1,2}$, Yoji Hirano, M.D., Ph.D. ${ }^{1}$, Itta Nakamura, M.D. ${ }^{1}$, Yuko Oda, M.D. ${ }^{1}$, Tomofumi Miura, M.D., Ph.D. ${ }^{1}$, Toshiaki Onitsuka, M.D., Ph.D. ${ }^{1}$

${ }^{1}$ Department of Neuropsychiatry, Graduate School of Medical Sciences, Kyushu University, Fukuoka, Japan

${ }^{2}$ Department of Rehabilitation, International University of Health and Welfare, Fukuoka, Japan

Corresponding Author: Dr. Toshiaki Onitsuka, Department of Neuropsychiatry, Graduate School of Medical Sciences, Kyushu University, 3-1-1 Maidashi, Higashiku, Fukuoka 812-8582, Japan.

TEL: +81-92-642-5627; FAX: +81-92-642-5644

E-mail: toshiaki@npsych.med.kyushu-u.ac.jp 


\begin{abstract}
Schizophrenia has been conceptualized by dysfunctional cognition and behavior related to abnormalities in neural circuitry. The functioning of the neural circuitry can be assessed using the auditory steady state response (ASSR). Moreover, in recent years, research on high (>60 Hz) gamma band oscillations has become of increasing interest. The current study used whole-head, 306-channel magnetoencephalography (MEG) and investigated low and high gamma band oscillations with the ASSR. The subjects comprised 17 patients with schizophrenia and 22 controls. The current study investigated the MEG-ASSR elicited by click trains of 20-, 30-, 40and $80-\mathrm{Hz}$ frequencies, and symptom-ASSR associations in patients with schizophrenia. The mean power, phase-locking factor, dipole moments and source locations of the ASSR were estimated. The main findings were: (1) patients with schizophrenia showed bilaterally reduced ASSR power and dipole moments specific to the $40-\mathrm{Hz}$ and $80-\mathrm{Hz}$ frequencies; (2) patients with schizophrenia showed less right-greater-than-left 40-Hz ASSR power and phase-locking factor compared with healthy subjects, indicating that schizophrenics may be characterized by an abnormal asymmetry of the 40-Hz ASSR; (3) increased severity of global hallucinatory experiences was significantly associated with smaller left 80-Hz MEG-ASSR in patients with schizophrenia. The current study highlights the high and low frequency gamma abnormalities and provides clear evidence that schizophrenia is characterized by abnormalities in neural circuitry.
\end{abstract}

Key Words: schizophrenia; magnetoencephalography; auditory steady state response; high frequency gamma synchronization; low frequency gamma synchronization 


\section{Introduction}

Since the time of Bleuler, schizophrenia had been described as "splitting of the psychic functions” (Bleuler, 1950/1911). More recently, schizophrenia has been conceptualized by a dysfunction in cognition and behavior related to abnormalities in neural circuitry. The functioning of the neural circuitry can be assessed using the auditory steady state response (ASSR). The ASSR seems to show resonant frequencies at around $40 \mathrm{~Hz}$ and $80 \mathrm{~Hz}$ with a larger power at $40 \mathrm{~Hz}$ (Picton et al., 2003). At these frequencies, the power of the ASSR is enhanced compared with that at other stimulation frequencies. In recent years, research on the ASSR has become of increasing interest, because the neural mechanisms responsible for the generation of synchrony have been revealed both in vivo and in vitro (e.g., Brenner et al., 2009; Uhlhaas et al., 2011). Moreover, both low (30-60Hz) and high $(>60 \mathrm{~Hz})$ gamma band oscillations can be generated by recurrent inhibition but differ in their relationship to the spiking activity of parvalbumin-containing interneurons, their pharmacological modulation profiles as well as their layer specificity (Oke et al., 2010). Magnetoencephalography (MEG) offers higher spatial resolution than electroencephalography (EEG) in accurately locating the position of neuronal activity. Moreover, MEG may be suitable to detect high gamma band oscillations because MEG waveforms cannot be deformed with conductivity affecting volume currents. In the present study, we used MEG to investigate low and high gamma band oscillations using the ASSR.

In terms of low gamma band oscillations, the ASSR and evoked/induced oscillations to auditory and visual stimuli have been extensively investigated. In schizophrenia, a reduced 40Hz-ASSR has been reported repeatedly. For example, Kwon et al. (1999) reported a reduced 40Hz-ASSR and showed delayed phase synchronization/desynchronization to a click train in schizophrenia. For evoked or induced oscillations, Spencer et al. (2004) demonstrated that visual 
gestalt stimuli led to abnormal evoked oscillatory activity around $40 \mathrm{~Hz}$ over the occipital lobe in schizophrenia, and that this abnormal evoked oscillatory activity was associated with visual hallucinations. In healthy subjects, Ross and colleagues (2005) reported a 40Hz-ASSR that was larger in the right than the left hemisphere, indicating that the left auditory cortex dominantly processes fast temporal changes in sound, whereas the right auditory cortex dominantly processes the spectral fine structure of the sound. They also suggested that the right auditory cortex may be more responsive to pitch processing and sound periodicity. Hamm and coworkers (2011) reported that schizophrenics showed smaller enhancements of the 40Hz-ASSR in their right hemispheres compared with healthy subjects. As noted before, it has been reported that schizophrenics show abnormal neuronal responses to sound periodicity. Moreover, it has been postulated that reduced or reversed brain asymmetries may play important roles in the pathology of schizophrenia (for example see Crow et al., 1989). Based on these findings, in the present study, we predicted that the patients would show reduced 40Hz-MEG-ASSRs and less right-greater-than-left $40 \mathrm{~Hz}$-ASSR compared with healthy subjects.

With respect to high gamma band oscillations, Grützner et al. (2010) used Mooney face stimuli and reported that distributed neural generators are involved in high gamma band oscillations for the processing of Mooney images in healthy subjects. There has been uniform agreement that MEG oscillations in the high gamma band are reliable markers of cortical activity during a variety of cognitive tasks (Kaiser et al., 2004). However, as far as we know, only one study has investigated the high gamma band ASSR in schizophrenia (Hamm et al., 2011). Although the ASSR itself may not reflect cognitive processes, the resonant frequencies of the ASSR suggest that basic neural circuits predominantly oscillate at these frequencies, and that the ASSR may shed light on the neural circuit functions of auditory-evoked and cognition-related 
gamma band oscillations. Therefore, in the present study, we also investigated high gamma band $(80 \mathrm{~Hz})$ MEG-ASSRs and the association between $80 \mathrm{~Hz}$-ASSRs and auditory hallucinations in schizophrenics, because $80 \mathrm{~Hz}-\mathrm{MEG}$-ASSRs may be more closely related to the function of cognition-related auditory neural circuitry than low gamma ASSRs.

\section{Methods}

\subsection{Subjects}

Seventeen (6 males, 11 females) schizophrenics and 22 healthy control subjects (9 males, 13 females) participated in this study. All subjects had normal hearing, were aged 20-63 years and were right-handed (Oldfield, 1971). After a complete description of the study, all participants signed an informed consent form according to the regulations of the Ethics Committee of the Graduate School of Medical Sciences, Kyushu University. The exclusion criteria were: 1) neurological illness or major head trauma; 2) electroconvulsive therapy; 3) alcohol or drug dependence; 4) alcohol or drug abuse within the past five years; or 5) a verbal intelligence quotient below 75. Healthy controls were screened using the Structured Clinical Interview (SCID)-non-patient edition. No healthy controls had an Axis-I psychiatric disorder themselves or in their first-degree relatives.

All patients were recruited from Kyushu University Hospital and were diagnosed based on the SCID-DSM IV and medical records. The patients were assessed on the Scale for the Assessment of Positive Symptoms (SAPS) (Andreasen, 1984) and the Scale for the Assessment of Negative Symptoms (SANS) (Andreasen, 1983). Demographic data for all subjects are presented in Table 1. All patients were receiving neuroleptic medication [typical neuroleptics (2/17 patients), atypical (12/17) or both (3/17)], with a mean daily dose equivalent to 
541ะ367mg of chlorpromazine (Woods, 2003).

\subsection{Stimuli}

The stimuli were 1-msec clicks, presented binaurally as trains of clicks for each stimulus (20, 30, 40 and $80 \mathrm{~Hz})$. The duration of each click train was $500 \mathrm{msec}$, and the intensity of the click trains was $80 \mathrm{~dB}$ sound pressure level. The inter-train interval was 500msec. The mean numbers of presented click trains in one block were 317.6 \pm 109.4 for healthy controls and $313.2 \pm 92.0$ for schizophrenic patients, and there was no significant group difference $(t[37]=0.14$, $p=0.89)$. The order of blocks was randomized across subjects.

\subsection{Data acquisition and processing}

The MEG signals were acquired using a whole-head, 306-channel sensor array (Vectorview; ELEKTA Neuromag, Helsinki, Finland). In this study, we analyzed MEG data recorded by the 22-channel, planar-type gradiometers around the sensor with maximum response for each hemisphere (Figure 1), based on previous studies (e.g., Palva et al., 2002; Teale et al., 2008; Shin et al., 2009). Prior to the recording, four head position indicator (HPI) coils were attached to the scalp, and a three-dimensional (3D) digitizer was used to measure the anatomical landmarks of the head with respect to the HPI coils. The precise location of the head with respect to the sensor array was determined using the HPI coils. The recording bandpass filter was $0.01-330 \mathrm{~Hz}$, and the sampling rate was $1 \mathrm{kHz}$. The subjects were instructed to keep their eyes open, remain attentive and listen to trains of clicks presented through the earphones. A spatiotemporal signal space separation (tSSS) method was applied off-line to the recorded raw data (Taulu et al., 2006). tSSS-reconstructed raw data with signal variations exceeding 4000fT were excluded. The data were averaged with the following conditions: the analysed period 
included 400ms before and 900ms after stimulus onset, and 200 responses were averaged for each type of stimulus.

\subsection{Frequency analysis}

We used an estimation of the time-frequency energy based on the wavelet transform of

the signal. The signal was convoluted by complex Morlet's wavelets $\omega\left(t, f_{0}\right)$ having a Gaussian shape with the wavelet being centered at the center frequency $f_{0}$ and time $t$ :

$\omega\left(t, f_{0}\right)=A \cdot \exp \left(-t^{2} / 2 \sigma^{2}\right) \cdot \exp \left(2 i \pi f_{0} t\right)$, where $2 \pi f_{0} \sigma=7$ in 1-Hz steps. Wavelets were normalized so that their total energy was 1 , the normalization factor $A$ being equal to $(\sqrt{2 \pi} \sigma)^{-1}$. We defined the squared modulus of the result of the convolution of a complex wavelet $\omega\left(t, f_{0}\right)$ with the averaged responses $s(t)$ : power $\left(t, f_{0}\right)=\left|\omega\left(t, f_{0}\right) \otimes s(t)\right|^{2}$ as the ASSR-power, where the symbol $\otimes$ indicates convolution. The square-root transform was applied to the ASSR-power for normalization. We also calculated the ASSR-phase-locking factor (PLF) using the following formula: $\operatorname{PLF}\left(t, f_{0}\right)=\frac{\left|\sum_{i} \frac{\omega(t, f) \otimes s(t)}{|\omega(t, f) \otimes s(t)|}\right|}{N}$. The PLF ranges from 0 (purely non-phase-locked activity) to 1 (strictly phase-locked activity). In calculating the power and PLF, we applied baseline correction (from -200 to -100msec). The mean power and PLF from $0-500$ msec for each stimulus were averaged across $10 \mathrm{~Hz}$ bands.

\subsection{Dipole moments and source localization}

The averaged responses were digitally filtered using a band-pass Butterworth filter (band pass; $15-25 \mathrm{~Hz}$ for $20 \mathrm{~Hz}$ stimulation, $25-35 \mathrm{~Hz}$ for $30 \mathrm{~Hz}, 35-45 \mathrm{~Hz}$ for $40 \mathrm{~Hz}, 75-85 \mathrm{~Hz}$ for $80 \mathrm{~Hz}$ ). A single moving equivalent current dipole source model was applied, and dipole fits in each hemisphere were calculated by a least-squares fit. Single source dipole localization was 
performed for each time-point for 0-500msec after stimulus onset. Only dipoles with goodness-of-fit criteria (>0.9) were chosen. The dipole locations were expressed by $x, y$, and z-coordinates.

T1-weighted 3T magnetic resonance imaging (MRI) (Philips Achieva, Best, The Netherlands) of 12 of 17 schizophrenics and 15 of 22 healthy control subjects was performed with a 1-mm slice thickness. To overlay the calculated dipoles onto the images of the subject's brain, the nasion and bilateral preauricular points were identified on the images by registering the fiduciary points and traced points over the scalp on the MRI-scanner.

\subsection{Statistical analysis}

The mean ASSR-powers, PLF and dipole moments analyzed using repeated measures analysis of variance (rmANOVA) with group (schizophrenia or control) as a between-subjects factor; and frequency (20,30, $40 \mathrm{or} 80 \mathrm{~Hz}$ ) and hemisphere (left or right) as within-subjects factors. For dipole locations, multivariate ANOVA (MANOVA) was performed with group as a between-subjects factor, and frequency, hemisphere and axis (x, y or $\mathrm{z}$ ) as within-subjects factors. Degrees of freedom were adjusted with the Huynh-Feldt epsilon for factors with more than two levels. Correlations between the severity of the auditory hallucinations and the ASSR-powers were evaluated using Spearman's rho. We considered all results to be significant at $p \leq 0.05$.

\section{Results}

\subsection{Demographics}

There were no significant group differences in demographic data except SES, consistent with reduced functioning due to this disorder (Table 1). In addition, there was no significant correlation between the medication dose and ASSR-power $(0.10 \leq$ rho $\leq 0.47,0.06 \leq p \leq 0.69)$. 


\subsection{Mean ASSR- power}

Figure 2 shows group averaged time-frequency maps of ASSR-power for each hemisphere. An rmANOVA demonstrated significant main effects of frequency $(F[3,35]=56.6$, $p<0.001)$ and hemisphere $(\mathrm{F}[1,37]=11.5, p=0.002)$, and significant frequency-by-group $(\mathrm{F}[3,35]=3.2, p=0.004)$ and hemisphere-by-group $(\mathrm{F}[1,37]=5.9, p=0.02)$ interactions, with no other significant main group effect or interactions $(0.06 \leq p \leq 0.16)$. To delineate the significant frequency-by-group interaction, group differences were compared with $t$-tests using the average power of both hemispheres for each frequency. Patients showed significantly reduced ASSR-power at $40 \mathrm{~Hz}(t[37]=2.4, p=0.02)$ and $80 \mathrm{~Hz}(t[37]=3.2, p=0.003)$, while no significant group differences were observed for $20 \mathrm{~Hz}(t[37]=-0.93, p=0.36)$ or $30 \mathrm{~Hz}(t[37]=0.88, p=0.39)$. To examine whether patients show reduced right-greater-than-left ASSR, we evaluated the lateralization index (LI) of the ASSR-power as $(L-R) /(|L|+|R|)$, with $L$ and $R$ being the values in the left and right hemisphere, respectively (Kircher et al., 2004). T-tests were performed for the LI of the ASSR-powers in each frequency. The patients showed significantly larger LIs $(-0.03 \pm 0.13$ for patients; $-0.13 \pm 0.18$ for controls; $t[37]=-2.0, p=0.05)$ in the $40 \mathrm{~Hz}$ frequency, indicating less right-greater-than-left 40Hz-ASSR-powers in schizophrenics. No significant group differences were observed for the $20 \mathrm{~Hz}(t[37]=-0.05, p=0.96), 30 \mathrm{~Hz}(t[37]=-1.5, p=0.15)$ or $80 \mathrm{~Hz}(t[37]=-0.88, p=0.38)$ frequencies.

\subsection{Mean ASSR-PLF}

Figure 3 shows group averaged time-frequency maps of ASSR-PLF for each hemisphere. An rmANOVA demonstrated significant main effects of frequency $(\mathrm{F}[3,35]=88.7, p<0.001)$, hemisphere $(\mathrm{F}[1,37]=14.2, p=0.001)$, and significant frequency-by-hemisphere-by-group $(\mathrm{F}[3,35]=3.3, p=0.05)$, hemisphere-by-group $(\mathrm{F}[1,37]=5.2, p=0.03)$, and 
frequency-by-hemisphere $(\mathrm{F}[3,35]=4.7, p=0.02)$ interactions, with no other significant main group effect or interactions $(0.36 \leq p \leq 0.39)$. To characterize the nature of the significant frequency-by-hemisphere-by-group interaction, two-factor rmANOVAs were performed with hemisphere as a within-subjects factor and group as a between-subjects factor for each frequency.

For $20 \mathrm{~Hz}$, there were no significant main effects ( $p=0.13$ for hemisphere; $p=0.89$ for group) or group-by-hemisphere interaction ( $p=0.56)$. For $30 \mathrm{~Hz}$, there was a significant main effect of hemisphere $(F[1,37]=12.0, p=0.001)$ with no significant group effect $(p=0.81)$ or group-by-hemisphere interaction $(p=0.20)$. For $40 \mathrm{~Hz}$, there was a significant main effect of hemisphere $(\mathrm{F}[1,37]=9.1, p=0.005)$ and group-by-hemisphere interaction $(\mathrm{F}[1,37]=5.4, p=0.03)$ with no significant group effect ( $p=0.27)$. To delineate the significant group-by-hemisphere interaction, post-hoc t-tests were performed for each hemisphere. There was no significant group difference in the left hemisphere $(t[37]=0.42, p=0.68)$ with a trend-level difference in the right hemisphere $(t[37]=1.8, p=0.08)$. For $80 \mathrm{~Hz}$, there was a significant main effect of group ( $\mathrm{F}[1,37]=5.9, p=0.02)$ with no significant hemisphere effect $(p=0.07)$ or group-by-hemisphere interaction $(\mathrm{F}[1,37]=1.6, p=0.22)$, indicating bilateral $80 \mathrm{~Hz}-\mathrm{PLF}$ reduction in schizophrenia. For the LI of the 40Hz-ASSR-PLF, patients showed significantly larger LI than controls $(-0.04 \pm 0.12$ for patients; $-0.13 \pm 0.13$ for controls; $t[37]=-2.1, p=0.04$ ), indicating less right-greater-than-left 40Hz-ASSR-PLF in schizophrenia.

\subsection{Dipole moments and locations}

Table 2 shows group mean dipole moments in each group. Patients showed significantly reduced dipole moments for $40 \mathrm{~Hz}(t[37]=4.1, p<0.001)$ and $80 \mathrm{~Hz}(t[37]=3.5, p=0.001)$ stimuli, while no significant group differences were observed for $20 \mathrm{~Hz}(t[37]=-1.1, p=0.26)$ and $30 \mathrm{~Hz}$ 
$(t[37]=1.8, p=0.09)$ stimuli.

For dipole locations, MANOVA demonstrated no group effect and no interactions related to group, indicating no significant group differences for dipole locations of the ASSR (see Table 3). Figure 4 shows the source locations of the ASSR for one healthy subject, projected onto the appropriate coronal MRI sections. The ASSRs were generated in or near the Heschl's gyrus.

\subsection{Correlations between the ASSR and demographic/clinical measurements}

In both groups, no significant correlations were observed between the ASSR variables and the demographic data $(0.08 \leq p \leq 0.95)$. Neuroleptic dose (chlorpromazine equivalent) did not significantly correlate with any ASSR variable in schizophrenia (0.0토 $p \leq 0.99)$.

There was a significant negative correlation between left $80 \mathrm{~Hz}$-ASSR-powers and auditory hallucination scores (rho=-0.504, $p=0.04$ ), while no significant negative correlations were observed between auditory hallucination scores and the other ASSR frequencies (Figure 5). Additionally, we compared the significances of the correlation coefficients between the left hemisphere responses to the $80 \mathrm{~Hz}$ stimulus and the left hemisphere responses to the other stimulation frequencies using Fisher's r-to-z transformations. Given the directional nature of our hypothesis, one-tailed tests were used. Based on Fisher's Z transformation, the $p$-values for the differences in the correlation coefficients were 0.03 between the 20 and $80 \mathrm{~Hz}(\mathrm{z}=1.88), 0.003$ between the 30 and $80 \mathrm{~Hz}(\mathrm{z}=2.71)$, and 0.13 between the 40 and $80 \mathrm{~Hz}(\mathrm{z}=1.11)$ frequencies.

\section{Discussion}

The current study investigated the MEG-ASSR elicited by click trains of 20, 30, 40 and $80 \mathrm{~Hz}$ frequencies, and symptom-ASSR associations in schizophrenia. The major findings of this study were: (1) patients showed bilaterally reduced ASSR-power and dipole moments, specific to 
the $40 \mathrm{~Hz}$ and $80 \mathrm{~Hz}$ frequencies; (2) patients showed less right-greater-than-left 40Hz-ASSR-power and PLF; (3) increased severity of global hallucinatory experiences was significantly associated with a smaller left 80Hz-MEG-ASSR in schizophrenia.

For high gamma band oscillations, Hamm et al. (2011) reported bilateral reduced 80Hz-ASSR in schizophrenia, and the current study supports their findings. Uhlhaas et al. (2011) concluded that high gamma band activity might be a fundamental aspect of temporal coding in cortical networks. Moreover, some studies have suggested that high gamma band activity is linked to higher cognitive functions (Ray et al., 2007). As noted before, the ASSR itself may not reflect cognitive processes; however, the $80 \mathrm{~Hz}$-ASSR may reflect the neural circuit functions of auditory-evoked and cognition-related high gamma band oscillations at least to some extent. The present study showed reduced $80 \mathrm{~Hz}-\mathrm{MEG}$-ASSR in schizophrenia, partially reflecting deficient neural circuitry of temporal coding in cortical networks.

The present study demonstrated that schizophrenics showed reduced 40Hz-ASSR-power and dipole moments. Several previous studies have reported reduced 40Hz-ASSR in patients and their relatives. For example, Kwon et al. (1999) reported that schizophrenia showed diminished 40Hz-ASSR-power. Hong et al. (2004) reported that relatives with schizophrenia spectrum personality symptoms had reduced 40Hz-ASSR-power. Light et al. (2006) reported reduction in both evoked power and phase synchronization in response to 30 and 40Hz-ASSR in schizophrenia. For the PLF of $40 \mathrm{~Hz}-\mathrm{ASSR}$, the present study showed no significant group difference in the left hemisphere, but a trend-level difference in the right hemisphere. However, Spencer et al. (2008) reported that PLF deficits in the 40Hz-ASSR were more pronounced over the left hemisphere in first-episode schizophrenia. They also reported that the PLF was reduced in schizophrenia for the right hemispheric tangential and the left hemispheric radial dipoles. Our 
current finding may not conflict completely with this, because MEG cannot detect the magnetic fields generated by a radial dipole with good detection of a tangential dipole. On the other hand, EEG can record the potentials of a radial dipole as well as a tangential dipole.

The current study also revealed less right-greater-than-left 40Hz-ASSR-power and PLF in schizophrenia. A right hemisphere dominance for 40Hz-ASSR has been reported in healthy subjects (Ross et al., 2005). In schizophrenia, Hamm et al. (2011) reported reduced 40Hz-ASSR in the right hemisphere. It has been postulated that reduced or reversed brain asymmetry may be importantly related to schizophrenia pathology (Crow et al., 1989). In schizophrenia, several studies have reported possible abnormal language lateralization (Delisi et al., 1992; Lennox et al., 1999; Dollfus et al., 2005.), abnormal event-related potential asymmetry (e.g., Onitsuka et al., 2009), and reduced or reversed anatomical brain volume asymmetry (e.g., Niznikiewicz et al., 2000). Moreover, our previous study (Hirano et al., 2008) reported that patients showed opposite hemispheric patterns to normal controls in evoked oscillatory activity. Schizophrenia pathophysiology may be characterized by reduced or reversed brain asymmetry neuroanatomically and neurophysiologically.

With respect to clinical correlations, increased severity of global hallucinatory experiences was significantly associated with smaller left $80 \mathrm{~Hz}-\mathrm{MEG}-\mathrm{ASSR}$ in patients. In a previous study of $80 \mathrm{~Hz}-\mathrm{ASSR}$, Hamm et al. (2011) reported that increased severity of negative symptoms was significantly associated with smaller 80Hz-MEG-ASSR in schizophrenia.

Recently, different mechanisms for the generation of low and high gamma band oscillations have been proposed. In this study, the association between $80 \mathrm{~Hz}-\mathrm{ASSR}$ and auditory hallucinations was substantially higher than that for the 20 or $30 \mathrm{~Hz}-\mathrm{ASSR}$. This finding may indicate different mechanisms generating low and high gamma band oscillations. 
In reviewing the current study, it is important to point out several possible limitations. First, the current study cannot answer the question of whether the abnormal ASSR pattern that was observed is associated with the progression of schizophrenia during the peri- and/or post-onset course of illness, or whether it is neurodevelopmental in origin, or perhaps a combination of both. Second, although no ASSR was significantly correlated with neuroleptic dosage, in the current study we did not exclude any effects of chronic treatment with neuroleptic medications on ASSR abnormalities in these patients. Third, the sample examined was predominantly female, and gender effects thus remain to be investigated. Finally, the association between reduced left hemisphere $80 \mathrm{~Hz}$-ASSRs and auditory hallucinations should be confirmed in a larger sample.

In summary, the current study highlighted the abnormal 80Hz-ASSR and provided clear evidence that schizophrenia is characterized by abnormalities in neural circuitry. 


\section{References}

Andreasen, N.C., 1983. The Scale for the Assessment of Negative Symptoms (SANS). Iowa City, IA: University of Iowa.

Andreasen, N.C., 1984. The Scale for the Assessment of Positive Symptoms (SAPS). Iowa City, IA: University of Iowa.

Bleuler, E., 1950/1911. Dementia praecox or the group of schizophrenias. New York: Intl. Univ. Press.

Brenner, C.A., Krishnan, G.P., Vohs, J.L., Ahn, W.Y., Hetrick, W.P., Morzorati, S.L., O'Donnell, B.F., 2009. Steady state responses: electrophysical assessment of sensory function in schizophrenia. Schizophr. Bull. 35 (6), 1065-1077.

Crow, T.J., Ball, J., Bloom, S.R., Brown, R., Bruton, C.J., Colter, N., Frith, C.D., Johnstone, E.C., Owens, B.G., Roberts, G.W., 1989. Schizophrenia as an anatomy of development of cerebral asymmetry. A postmortem study and a proposal concerning the genetic basis of the disease. Arch. Gen. Psychiatry 46 (12), 1145-1150.

Delisi, L.E., Sakuma, M., Kushner, M., Finer, D.L., Hoff, A.L., Crow, T.J., 1997. Anomalous cerebral asymmetry and language processing in schizophrenia. Schizophr. Bull. 23 (2), 255-271.

Dollfus, S., Razafimandimby, A., Delamillieure, P., Brazo, P., Joliot, M., Mazoyer, B., Tzourio-Mazoyer, N., 2005. Atypical hemispheric specialization for language in right-handed schizophrenia patients. Biol. Psychiatry 57 (9), 1020-1028.

Grützner, C., Uhlhaas, P.J., Genc, E., Kohler, A., Singer, W., Wibral, M., 2010. Neuroelectromagnetic correlates of perceptual closure processes. J. Neurosci. 30 (24), 
8342-8352.

Hamm, J.P., Gilmore, C.S., Picchetti, N.A., Sponheim, S.R., Clementz, B.A., 2011.

Abnormalities of neuronal oscillations and temporal integration to low- and

high-frequency auditory stimulation in schizophrenia. Biol. Psychiatry in press.

Hirano, S., Hirano, Y., Maekawa, T., Obayashi, C., Oribe, N., KurHoki, T., Kanba, S.,

Onitsuka, T., 2008. Abnormal neural oscillatory activity to speech sounds in

schizophrenia: a magnetoencephalography study. J. Neurosci. 28 (19), 4897-4903.

Hong, L.E., Summerfelt, A., McMahon, R., Adami, H., Francis, G., Elliott, A., Buchanan,

R.W., Thaker, G.K., 2004. Evoked gamma band synchronization and the liability for schizophrenia. Schizophr. Res. 70 (2-3), 293-302.

Kaiser, J., Bühler, M., Lutzenberger, W., 2004. Magnetoencephalographic gamma-band responses to illusory triangles in humans. Neurioimage 23 (2), 551-560.

Kircher, T.T., Rapp, A., Grodd, W., Buchkremer, G., Weiskopf, N., Lutzenberger, W., Ackermann, H., Mathiak, K., 2004. Mismatch negativity responses in schizophrenia: a combined fMRI and whole-head MEG study. Am. J. Psychiatry 161 (2), 294-304.

Kwon, J.S., O’Donell, B.F., Wallenstein, G.V., Greene, R.W., Hirayasu, Y., Nestor, P.G., Hasselmo, M.E., Potts, G.F., Shenton, M.E., McCarley, R.W., 1999. Gamma frequency-range abnormalities to auditory stimulation in schizophrenia. Arch. Gen. Psychiatry 56 (11), 1001-1005.

Lennox, B.R., Park, S.B., Jones, P.B., Morris, P.G., 1999. Spatial and temporal mapping of neural activity associated with auditory hallucinations. Lancet 353 (9153), 644.

Light, G.A., Hsu, J.L., Hsieh, M.H., Meyer-Gomes, K., Sprock, J., Swerdlow, N.R., Braff, D.L., 2006. Gamma band oscillations reveal neural network cortical coherence 
dysfunction in schizophrenia patients. Biol. Psychiatry 60 (11), 1231-1240.

Niznikiewicz, M., Donnino, R., McCarley, R.W., Nestor, P.G., losifescu, D.V., O’Donnell, B., Levitt, J., Shenton, M.E., 2000. Abnormal angular gyrus asymmetry in schizophrenia. Am. J. Psychiatry 157 (3), 428-437.

Oke, O.O., Magony, A., Anver, H., Ward, P.D., Jiruska, P., Jefferys, J.G., Vreugdenhil, M., 2010. High-frequency gamma oscillations coexist with low-frequency gamma oscillations in the rat visual cortex. Eur. J. Neurosci. 31 (8), 1435-1445.

Oldfield, R.C., 1971. The assessment and analysis of handedness: the Edinburgh inventory. Neuropsychologia 9 (1), 97-113.

Onitsuka, T., Spencer, K.M., Lucia, L.C., Shenton, M.E., McCarley, R.W., Niznikiewicz, M.A., 2009. Abonormal asymmetry of the N170 repetition effect in male patients with Chronic schizophrenia. Brain Imaging Behav. 3, 240-245.

Palva, S., Palva, J.M., Shtyrov, Y., Kujala, T., Ilmoniemi, R.J., Kaila, K., Näätänen, R., 2002. Distinct gamma-band evoked responses to speech and non-speech sounds in humans. J. Neurosci. 22 (4), RC211.

Picton, T.W., John, M.S., Dimitrijevic, A., Purcell, D., 2003. Human auditory steady-state responses. Int. J. Audiol. 42 (4), 177-219.

Ray, S., Niebur, E., Hsiao, S.S., Sinai, A., Crone, N.E., 2007. High-frequency gamma activity $(80-150 \mathrm{~Hz})$ is increased in human cortex during selective attention. Clin. Neurophysiol. 119 (1), 116-133.

Ross, B., Herdman, A.T., Pantev, C., 2005. Right hemispheric laterality of human $40 \mathrm{~Hz}$ auditory steady-state responses. Cerebral Cortex 15 (12), 2029-2039.

Shin, K.S., Kim, J.S., Kang, D.H., Koh, Y., Choi, J.S., O’Donell, B.F., Chung, C.K., Kwon, J.S., 
2009. Biol. Psychiatry 65 (12), 1071-1078.

Spencer, K.M., Nestor, P.G., Perlmutter, R., Niznikiewicz, M.A., Klump, M.C., Frumin, M., Shenton, M.E., McCarley, R.W., 2004. Neuronal synchrony indexes disordered perception and cognition in schizophrenia. Proc. Natl. Acad. Sci. USA 101 (49), 17288-17293.

Spencer, K.M., Salisbury, D.F., Shenton, M.E., McCarley, R.W., 2008. Gamma-band auditory steady-state responses are impaired in first episode psychosis. Biol. Psychiatry 64 (5), 369-375.

Spencer, K.M., Niznikiewicz, M.A., Nestor, P.G., Shenton, M.E., McCarley, R.W., 2009. Left auditory cortex gamma synchronization and auditory hallucination symptoms in schizophrenia. BMC Neurosci. 10, 85.

Teale, P., Collins, D., Maharajh, K., Rojas, D.C., Kronberg, E., Reite, M., 2008. Cortical source estimates of gamma band amplitude and phase are different in schizophrenia. Neuroimage 42 (4), 1481-1489.

Taulu, S., Simola, J., 2006. Spatiotemporal signal space separation method for rejecting nearby interference in MEG measurements. Phys. Med. Biol. 51 (7), 1759-1768.

Uhlhaas, P.J., Pipa, G., Neuenschwander, S., Wibral, M., Singer, W., 2011. A new look at gamma? High- ( $>60 \mathrm{~Hz}) \gamma$-band activity in cortical networks: Function, mechanisms and impairment. Prog. Biophys. Mol. Biol. 105 (1-2), 14-28.

Woods, S.W., 2003. Chlorpromazine equivalent doses for the newer atypical antipsychotics. J Clin Psychiatry. 64 (6), 663-667. 


\section{Figure 1}

Layout of the measured channels. The MEG signals were acquired using a whole-head, 306-channel sensor array that comprises 102 identical triple-sensor elements. Each sensor consists of two orthogonal planar-type gradiometers and one magnetometer. We used 11 sensors (22-channel orthogonal gradiometers) around the maximum response in each hemisphere. Circled points indicate the sensors used for the analysis.

\section{Figure 2}

Group averaged time-frequency maps of ASSR-power for each hemisphere. The color scales signify ASSR-power.

\section{Figure 3}

Group averaged time-frequency maps of ASSR-PLF for each hemisphere. The color scales signify ASSR-PLF value.

HC, healthy controls; SZ, patients with schizophrenia.

\section{Figure 4}

Locations of the ASSR sources in one healthy subject, projected onto the appropriate subject's coronal MRI sections. The locations of the ASSR sources are shown for $20 \mathrm{~Hz}$ (red circles), $30 \mathrm{~Hz}$ (green), $40 \mathrm{~Hz}$ (blue) and $80 \mathrm{~Hz}$ (yellow). The right panel is $2 \mathrm{~mm}$ posterior to the left one. For 20Hz-ASSR, the coordinates are (in $\mathrm{mm}$ ): $\mathrm{x}=-41.2, \mathrm{y}=-0.9, \mathrm{z}=44.5$ in the left hemisphere; $\mathrm{x}=40.8$, $\mathrm{y}=-3.2, \mathrm{z}=49.6$ in the right. For $30 \mathrm{~Hz}-\mathrm{ASSR}$, the coordinates are $\mathrm{x}=-37.2, \mathrm{y}=-0.8, \mathrm{z}=42.3$ in the left; $\mathrm{x}=42.4, \mathrm{y}=-0.3, \mathrm{z}=49$ in the right. For $40 \mathrm{~Hz}-\mathrm{ASSR}$, the coordinates are $\mathrm{x}=-46.7, \mathrm{y}=-2.2$, 
$\mathrm{z}=35.2$ in the left; $\mathrm{x}=45.8, \mathrm{y}=-2.3, \mathrm{z}=48.5$ in the right. For $80 \mathrm{~Hz}$-ASSR, the coordinates are $x=-46.1, y=-1.1, z=37$ in the left; $x=60.7, y=-5.9, z=49.6$ in the right.

\section{Figure 5}

Scattergrams between the mean $80 \mathrm{~Hz}$ ASSR-powers in each hemisphere and the scores for auditory hallucination on the Scale for the Assessment of Positive Symptoms (SAPS). 